\title{
Diabetes y embarazo - Actualización
}

\author{
Orlando Restrepo Ochoa*
}

\section{RESUMEN}

Se hace una revisión que incluye la nueva clasificación etiológica y los nuevos criterios diagnósticos de la Diabetes Mellitus (DM). Igualmente, se analiza el metabolismo de la mujer embarazada diabética, los efectos del embarazo sobre la DM y viceversa.

Finalmente, se hace énfasis en el manejo metabólico de la DM durante el embarazo, trabajo de parto y parto; además, se discute el manejo obstétrico, incluyendo la vía del parto y el momento del mismo.

PALABRAS CLAVES: diabetes, insulinodependiente, hiperglicemia, hipoglicemia, macrosomía, trabajo de parto, parto.

\section{SUMMARY}

I make a revision that include the new etiologic classification and the new diagnostic criteria of Diabetes Mellitus (DM). Equality, I analyze the metabolism of the obstetric pregnant woman, the effects of gestation on DM and vice versa.

Finally, I emphasize in the metabolic management of the DM during antepartum period, labor and delivery; desides, I discuss the obstetric management, including route and timing of delivery.

KEY WORDS: diabetes, insulin-dependent, hyperglicemia, hypoglicemia, macrosomia, labor, delivery.

\section{Introducción}

La Diabetes Mellitus (DM) comprende un grupo de desórdenes metabólicos que se caracterizan por presentar altas concentraciones de glucosa plasmática como resultado de una insuficiente secreción de insulina, total o parcial, y/o por una resistencia a la acción de la misma. La hiperglicemia crónica se asocia con daño a largo plazo de varios órganos, especialmente riñones, ojos, nervios, corazón y vasos sanguíneos.

La DM es considerada como la entidad metabólica más común durante la gestación, presentándose más o menos en el $5 \%$ de los embarazos. El 90\% de estas pacientes presentan Diabetes Mellitus Gestacional (DMG), o sea mujeres con predisposición genética o metabólica a la diabetes, incapaces de compensar adecuadamente los efectos diabetógenos del embarazo; el restante $10 \%$ está conformado por mujeres con diabetes ya diagnosticadas antes del embarazo (DM1, DM2 y otros tipos) (1).

Los recursos modernos en el manejo de la DM (automonitoreo de la glucosa en casa, las bombas de insulina de aplicación subcutánea continua, los múltiples tipos de insulina, el ultrasonido y el monitoreo biofísico fetal), han revolucionado el resultado de las gestaciones complicadas con

Profesor de Grupo de Perinatología. Departamento de Obstetricia y Ginecología, Facultad de Medicina. Universidad de Antioquia este desorden relativamente común. Antes del descubrimiento de la insulina por Banting y Best en 1922, la culminación probable del embarazo era la muerte tanto de la madre como del feto; con la utilización de dicha hormona disminuyeron de manera impresionante los riesgos para la gestante y su producto.

En 1939, Priscilla White y col (2) reportan el manejo exitoso de 245 embarazadas diabéticas y encuentran que la incidencia de cetoacidosis diabética materna había disminuido dramáticamente y que la frecuencia de mortinatos era la mitad de la reportada en el período preinsulina.

Las siguientes tres décadas mostraron que el resultado de la gestación estaba estrechamente relacionado con el control metabólico; durante este período, las tasas de mortalidad perinatal cayeron del 35 al 10\%.

En 1970 se introduce el manejo interdisciplinario de la gestante diabética; esto y las nuevas tecnologías como el monitoreo biofísico fetal, la valoración de crecimiento fetal por ecografía y el análisis de la madurez pulmonar fetal en el líquido amniótico (LA), reducen notablemente la morbilidad neonatal. Sin embargo, persisten problemas mayores en el manejo de la madre y el feto. Aunque las tasas de mortinatos han disminuido notablemente, se cuestionan lo costoso de las pruebas biofísicas y las altas tasas de nacimientos pretérmino y de operación cesárea. Las anomalías congénitas continúan siendo 7-15 veces más frecuentes en las gestantes diabéticas que en las 
embarazadas sin la enfermedad y la macrosomía y el trauma al nacimiento ocurren con una frecuencia 10 veces mayor en los fetos diabéticos. $(1,3)$.

Nuestro conocimiento de la interrelación de la dieta, la actividad física, el estrés, los niveles de glucosa y el bienestar fetal, continúa siendo rudimentario y somos incapaces de prevenir la macrosomía y el trauma al nacimiento sin inaceptables altas tasas de cesárea (3).

\section{Metabolismo de los hidratos de carbono ( $\mathrm{H}$ de $\mathrm{C}$ ) durante el embarazo}

El embarazo es diabetógeno, entre otros por los siguientes factores: (4)

Resistencia a la insulina: el factor más importante es el progresivo aumento de la resistencia a la insulina que se produce durante la gestación. Esta insulino-resistencia surge como resultado del efecto hiperglicemiante de las hormonas de contrarregulación secretadas por la placenta y trata de vencerse mediante el aumento de la secreción de insulina, produciéndose una hiperinsulinemia concomitante. Igualmente, hay aumento de la destrucción de la insulina por el riñón y las insulinasas placentarias.

Aumento de la lipólisis: la madre utiliza la grasa para cubrir sus necesidades y guarda la glucosa para cubrir las del feto.

Cambios en la gluconeogénesis: el feto emplea alanina y otros aminoácidos, privando a la madre de los principales substratos para la gluconeogénesis. Ahora, este consumo de glucosa y aminoácidos por parte del feto explica la tendencia a la hipoglicemia en ayunas durante el embarazo (1).

Resumiendo, el metabolismo de los $\mathrm{H}$ de $\mathrm{C}$ durante la gestación se caracteriza por:

- Tendencia a la hipoglicemia y a la cetosis en ayunas

Tendencia a la hiperglicemia posprandial

Hiperinsulinemia e insulinorresistencia

\section{Efectos del embarazo sobre la diabetes}

El embarazo crea una pesada carga para la diabetes. Estas pacientes tienen una tendencia a la inestabilidad metabólica y requieren monitorización frecuente y un manejo estricto. Por tanto, son necesarias dosis mayores de insulina para lograr el control metabólico; hay progresión de la retinopatía y la nefropatía diabéticas y mayor riesgo de muerte en pacientes con miocardiopatía diabética $(1,4)$.

\section{Efectos de la diabetes mellitus sobre el embarazo}

La intolerancia a los $\mathrm{H}$ de $\mathrm{C}$ durante el embarazo es asintomática en la mayoría de los casos y produce un aumento significativo en la morbilidad maternofetal. Las consecuencias para el feto son más graves que las maternas. De ellas la más importante es la macrosomía, debido a la dificultad de su diagnóstico y a las controversias que existen con relación a su manejo (ver tabla 1).(4-5)

\section{Criterios de clasificación}

Priscilla White describe su clasificación pronóstica basándose en la edad del comienzo, la duración del tras-
Tabla 1

EFECTOS DE LA DIABETES SOBRE EL EMBARAZO

1. COMPLICACIONES MATERNAS

- Preeclampsia - eclampsia (afecta $10-25 \%$ de las gestantes diabéticas)

Infecciones (mayor incidencia de corioamnionitis, infección urinaria, cérvicovaginitis e infección puerperal).

- Hemorragia posparto (mayor incidencia por excesiva distensión)

- Parto por cesárea: altas tasas en gestantes diabéticas

\section{COMPLICACIONES FETALES}

Anomalías congénitas

Macrosomía-R.C.I.U.

Parto traumático

Prematurez y enfermedad de la membrana hialina Apnea y bradicardia

Alteraciones hematológicas y metabólicas del recién nacido (hipoglicemia, hipocalcemia, poliglobulia, hiperbilirrubinemia, síndrome de hiperviscosidad).

torno y las complicaciones. El defecto más grande de esta clasificación es que no contempla que la inestabilidad metabólica es el principal factor pronóstico en la gestante diabética insulinodependiente. En 1985, Freinkel modifica la clasificación de White, así: (1)

Clases:

A: diabetes gestacional

A1: glicemia en ayunas $<105 \mathrm{mg} / \mathrm{dl}$ (normal)

A2: glicemia en ayunas entre 105 y 129 mg/dl (intolerante)

B: glicemia en ayunas $>130 \mathrm{mg} / \mathrm{dl}$

B1: si el diagnóstico es de primera vez (DMG)

B2: inicio después de los 20 años y evolución menor de 10 años, persistiendo entre los embarazos (DM2)

Clases C-D-F-H-R: corresponden a los DM1 y DM2.

La clasificación publicada por el National Diabetes Data Group (NDDG) (6) y refrendada por la Organización Mundial de la Salud (OMS) (7) en 1980 y 1985 se basaba en una combinación de manifestaciones clínicas, requerimientos de tratamiento y patogénesis. Ella consideraba dos tipos de pacientes: las que conformaban la categoría clínica y las que se ubicaban en una categoría de riesgo estadístico.

En 1995, bajo el patrocinio de la Asociación Americana de Diabetes (ADA), se creó un Comité Internacional de expertos, el cual propone una serie de modificaciones al anterior esquema de clasificación, basado en la revisión de la literatura ya la luz de los nuevos conocimientos (ver tabla 2).(8).

Recordar que si solo nos basamos en ellos, aproximadamente del 35 al $50 \%$ de las diabéticas gestacionales se quedarán sin diagnosticar $(1,4)$. 
Tabla 2

\section{CLASIFICACION ETIOLOGICA DE LA DIABETES MELLITUS}

\section{DIABETES TIPO 1}

(Destrucción de la célula $\beta$, llevando a la deficiencia absoluta de insulina)

A. Mediada inmunológicamente

B. Idiopática

II. DIABETESTIPO 2

(Puede ir desde resistencia a la insulina con relativo déficit hasta predominancia del déficit)

\section{OTROSTIPOSESPECÍFICOS}

A. Defectos genéticos en la función de las células $B$

1. Cromosoma $12, \mathrm{HNF}-1 \alpha$ (antes MODY 3 )

2. Cromosoma 7, glucoquinasa (antes MODY 2)

3. Cromosoma 20, HNF - $4 \alpha$ (antes MODY 1)

4. DNA Mitocondrial

5. Otros

B. Defectos genéticos en la acción de la insulina

1. Resistencia a la insulina tipo A

2. Leprechaunismo

3. Síndrome de Rabson - Mendelhall

4. Diabetes lipoatrófica

5. Otros

C. Enfermedades del páncreas exocrino

1. Pancreatitis

2. Trauma - pancreatectomía

3. Neoplasia

4. Fibrosis quística

5. Hemocromatosis

6. Pancreatopatía fibrocalculosa

7. Otros

D. Endocrinopatías

1. Acromegalia

2. Síndrome de Cushing

3. Glucagonoma

4. Feocromocitoma

5. Hipertiroidismo

6. Somatostatinoma

7. Aldosteronoma

8. Otros

E. Inducida por fármacos o químicos

1. Vacor

2. Pentamidina

3. Ácido nicotínico

4. Glucocorticoides

5. Hormona tiroidea

6. Diazóxido

7. Agonistas $B$ - adrenérgicos

8. Tiazidas

9. Dilantén

10. $\alpha$-interferón

11. Otros
F. Infecciones

1. Rubéola congénita

2. Citomegalovirus

3. Otros

G. Formas poco frecuentes de diabetes mediadas inmunológicamente

1. Síndrome de "Stiff man" (hombre rígido)

2. Anticuerpos anti-receptor de insulina

3. Otros

H. Otros síndromes genéticos asociados algunas veces con diabetes

1. Síndrome de Down

2. Síndrome de klinefelter

3. Síndrome de Turner

4. Síndrome de Wolfram

5. Ataxia de Friedreich

6. Corea de Huntington

7. Síndrome de Lawrence Moon Beidel

8. Distrofia miotónica

9. Porfiria

10. Síndrome de Prader Willi

11. Otros

IV. DIABETES MELLITUS GESTACIONAL

Como puede apreciarse, los términos insulinodependiente y no insulinodependiente y sus acrónimos (DMID y DMNID) desaparecen, ya que ellos se refieren más a tipos de tratamiento que a etiología. Los términos tipo 1 y tipo 2 se retienen, pero en números arábigos.

El estado de disminución de la tolerancia a la glucosa es retenido y se añade uno nuevo, alteración de la glucosa en ayunas, el cual comparte las mismas características. Estos términos se refieren a un estado metabólico intermedio entre la homeostasis normal de la glucosa y la diabetes. Estos individuos son normoglicémicos en las actividades diarias, con valores normales de $\mathrm{HbAlC}$, pero ante un estímulo mayor reaccionan con hiperglicemia.

La DMG se conserva como fue definida por la OMS y el NDDG, pero ahora se recomienda la tamización selectiva y no universal para la intolerancia a la glucosa en pacientes embarazadas.

\section{Diabetes Mellitus 1 (DMI):}

1. Mediada inmunológicamente: resulta de la destrucción de la célula $B$ del páncreas mediada inmunológicamente. Los marcadores de la destrucción inmune incluyen los autoanticuerpos contra los islotes (ICAs), autoanticuerpos contra la insulina (IAAs), autoanticuerpos contra la descarboxilasa del ácido glutámico (GAD65) y los autoanticuerpos contra las fosfatasas de la tirosina (IA - 2 e IA - 2B). Uno o más de estos anticuerpos están presentes en el $85-90 \%$ de las pacientes cuando se detecta inicialmente la hiperglicemia en ayunas. 
Igualmente, la DM1 se asocia fuertemente con el HLA, específicamente con los genes DQA y DQB y es influenciada por los genes DRB. Estos alelos HLA - DR/ DQ pueden ser predisponentes o protectores. Aunque las pacientes con DM1 rara vez son obesas, la presencia de obesidad no es incompatible con el diagnóstico.

2. DM1 idiopática: algunas formas raras de DM1 notienen etiología autoinmune, tiene tendencia a la cetoacidosis y se presentan en pacientes de origen africano o asiático; además, tienen asociación familiar y no tienen marcadores inmunológicos.

\section{Diabetes Mellitus 2 (DM2):}

Estas pacientes presentan en su etiología un factor de resistencia a la acción de la insulina acompañado de cierto grado de disminución en la secreción de insulina, pudiendo predominar cualquiera de los dos estados. La etiología no está muy bien definida, no hay factores inmunes, la gran mayoría son obesos (la obesidad de por sí es causa de algún grado de resistencia a la insulina), existe asociación familiar, presentan niveles de insulina elevados o normales, pero insuficientes para los niveles de glucosa. El riesgo de presentarla aumenta con la edad, la obesidad, sedentarismo, antecedentes de DMG, hipertensión, dislipidemias y en ciertos grupos étnicos. Además, se asocia con una fuerte predisposición genética, más aún que la DM1 autoinmune.

\section{Diabetes Mellitus Gestacional (DMG):}

Se define como la intolerancia a los $\mathrm{H}$ de $\mathrm{C}$ de varios grados de severidad con aparición o primer reconocimiento durante el embarazo. La definición se aplica sin tener en cuenta si para el tratamiento se utiliza insulina o solo modificación de la dieta o si la condición persiste después del embarazo. Además, no excluye la posibilidad de que una intolerancia a la glucosa haya precedido o haya comenzado en forma concomitante con el embarazo (8-9).

La DMG complica más o menos el $4 \%$ de los embarazos en los Estados Unidos; sin embargo, la prevalencia varía entre el 1 y $14 \%$, dependiendo de la población estudiada.
La DMG puede repetir en el $15-30 \%$ de las pacientes; igualmente, el $47 \%$ de las mujeres que sufren DMG y que pertenecen al grupo étnico hispano latinoamericano, desarrollan DM2 después de cinco años (10).

Pruebas para DMG: O'Sullivan y Mahan en 1964 proponen los criterios para la intolerancia a la glucosa, basados en los datos obtenidos de pruebas de tolerancia oral a la glucosa (PTOG) en 752 mujeres embarazadas. Ahora, en 1979, (6) el NDDG revisa estos criterios y convierte los valores de sangre total a valores en plasma. Estos criterios fueron adoptados por la ADA y por el Colegio Americano de Obstetras y Ginecólogos (ACOG), (12) pero tienen variaciones con respecto a los criterios de la OMS.

Hay un consenso general que la prevalencia de la DMG está aumentando mundialmente, pero hay considerable controversia acerca de la importancia clínica de la DMG y de la magnitud de su impacto en la madre y su hijo (9). Dado que la DMG es asintomática, solamente la búsqueda insistente del estado bioquímico con relación al metabolismo de los $\mathrm{H}$ de $\mathrm{C}$, nos llevará a mayores tasas de diagnóstico precoz de DMG.

Son factores de riesgo para DMG: (1)

- Obesidad o sobrepeso materno

- Historia familiar de DM en primera línea

- Antecedentes de mortinatos o de muerte neonatal inexplicable

- Antecedentes de macrosómicos (>4.000 gramos)

- Antecedentes de parto traumático con o sin trastorno neurológico asociado en el niño

Antecedentes de anomalías congénitas

- Prematuridad o hidramnios previos

- HTA o antecedentes de preeclampsia-eclampsia

Infecciones urinarias recurrentes

Antecedentes de DMG o de intolerancia a los $\mathrm{H}$ de $\mathrm{C}$

Glucosuria persistente

Candidiasis severa recurrente

Pobre historia reproductiva

Trastornos tiroideos (hipertiroidismo)

Edad $>25$ años

- Grupos étnicos de alto riesgo
En el resumen y recomendaciones de las anteriores conferencias internacionales de trabajo en diabetes gestacional, (13-15) la tamización para DMG fue recomendada en todas las gestaciones, con la premisa sana de que la DMG, es una de las pocas entidades en donde con mayor claridad se puede realizar medicina preventiva. La tamización universal es todavía recomendada para mujeres de grupos étnicos con relativamente altas tasas de intolerancia a los $\mathrm{H}$ de $\mathrm{C}$ durante el embarazo y de DM más tarde en su vida. Esto incluye mujeres de ancestro hispano, africano, nativos americanos, del sur o del oriente asiáticos, islas del pacífico o indígenas con ancestro australiano, particularmente cuando ellos residen en países occidentales o en ambientaciones urbanas. Contrariamente, ciertas características son de bajo riesgo para DMG y desde el punto de vista costobeneficio no es importante tamizar a estas pacientes. Aquellas con bajo riesgo incluyen:

Mujeres que no son miembros de grupos étnicos con riesgo elevado para desarrollar DM2. 
- Quienes no tienen historia previa de tolerancia anormal a la glucosa o resultados obstétricos pobres usualmente asociados a DMG, y

- Quienes tienen todas las características siguientes: edad $<25$ años, peso corporal normal y no historia familiar de DM.

Esta tamización tiene una sensibilidad que va del 75 al $85 \%$ y una especificidad que va del 85 al $90 \%$, según la serie estudiada (1). Consiste en administrar en la primera consulta prenatal (antes de la semana 20), 50 gramos de glucosa y determinar la glicemia plasmática una hora después (descartar DM pregestacional); no es necesario que la paciente esté en ayunas y se puede realizar a cualquier hora del día. Si el resultado es normal, dicha tamización se repetirá entre las 24-28 semanas de gestación, tiempo en el cual se presenta el máximo efecto diabetógeno del embarazo. Un valor $\geq 140 \mathrm{mg} / \mathrm{dl}$ (o $\geq 135$ $\mathrm{mg} / \mathrm{dl}$ si se ayunó durante la noche) requiere un estudio completo con una PTOG con $100 \mathrm{~g}$ (ver tabla 3) (8).

El diagnóstico de DMG se hace con dos o más valores superiores a los mencionados en la tabla 3; cuando hay un solo valor alterado, la prueba es considerada patológica, presentándose riesgo de complicaciones como la macrosomía fetal y la preeclampsia-eclampsia. Ahora, el ACOG recomienda que en poblaciones con alta prevalencia de DMG o con francos factores de riesgo para la misma, debe hacerse directamente la PTOG con 100g, sin tamización previa (12).

Por otra parte, se han hecho propuestas para cambiar el esquema diagnóstico antes anotado. Es así como Carpenter y Coustan (16) proponen puntos de corte para la glucosa plasmática que parecen representar con mayor exactitud las determinaciones originales de O'Sullivan y Mahan; en otras palabras, dichas extrapolaciones producen valores más bajos para concentraciones anormales de glucosa en plasma y aumentan el número de gestaciones definidas como DMG. Además, estudios adicionales han sido llevados a cabo utilizando $75 \mathrm{~g}$ de carga, como lo recomienda la OMS, con resultados similares (17). En la tabla 4, podemos observar los valores para dichas PTOG: (9)

Igualmente, el diagnóstico de DMG se hace con dos o más valores superiores a los anotados. Cualquiera de estos test diagnósticos con $100 \mathrm{~g} 075 \mathrm{~g}$ es aceptable, pero su sensibilidad (precisión diagnóstica) y especificidad deben seguir siendo evaluados (9).

A las seis semanas posparto, la paciente debe ser sometida a una PTOG con $75 \mathrm{~g}$, tomando muestras en ayunas y

Tabla 3 ESQUEMA PARA LA TAMIZACIÓN Y EL
DIAGNÓSTICO DE DMG

\begin{tabular}{|ccc|}
\hline $\begin{array}{c}\text { Glucosa } \\
\text { plasmática }\end{array}$ & $\begin{array}{c}\text { Tamización } \\
\text { con } \mathbf{5 0} \mathbf{g}\end{array}$ & $\begin{array}{c}\text { Prueba } \\
\text { diagnóstica con } \mathbf{1 0 0} \mathbf{g}\end{array}$ \\
\hline Ayunas & & $105 \mathrm{mg} / \mathrm{dl}$ \\
1 hora & & $190 \mathrm{mg} / \mathrm{dl}$ \\
2 horas & $135-140 \mathrm{mg} / \mathrm{dl}$ & $165 \mathrm{mg} / \mathrm{dl}$ \\
3 horas & & $145 \mathrm{mg} / \mathrm{dl}$ \\
\hline
\end{tabular}

dos horas posprandial, para ser reclasificada como: 1) diabetes, 2) intolerancia a la glucosa en ayunas, 3) intolerancia a la glucosa o 4) normoglicemia, de acuerdo con los nuevos criterios para el diagnóstico de DM (ver tabla 5). Se toman las seis semanas posparto, ya que en este tiempo los receptores insulínicos normalizan su afinidad y densidad (1). No olvidar que en la mayoría de los casos de DMG, la regularización de la glucosa regresa a su estado normal después del parto. Aquellas pacientes consideradas como normoglicémicas en este tiempo, deben ser evaluadas para diabetes como mínimo cada año (1, 8-9).

Nuevos criterios diagnósticos para DM: los criterios diagnósticos para DM recomendados por el NDDG y por la OMS, (6-7) también fueron revisados y modificados por la ADA; para su evaluación se tomaron una serie de estudios epidemiológicos que relacionaban la glicemia en ayunas, la glicemia dos horas posprandial y los niveles de HbA1C (ver tabla 5) (8).

\section{Manejo de la diabetes mellitus durante el embarazo}

Las diferencias en el pronóstico materno-fetal hace necesario distinguir dos tipos de mujeres embarazadas con diabetes: (1)

$6.1 \mathrm{La}$ mujer diabética que se embaraza (DM pregestacional). Dentro de esta categoría existen dos grupos:

- Paciente sin complicaciones vasculares crónicas: aquí el buen control metabólico permite que el embarazo curse sin complicaciones.

- Pacientes con complicaciones vasculares crónicas: la gestación acelera el desarrollo microangiopático a nivel renal y retiniano.

6.2 Diabetes mellitus gestacional: aquí el trastorno metabólico acaba de instaurarse, el riesgo de un mal control metabólico recae sobre el feto. Los reportes indican que cuando la DMG es diagnosticada y tratada intensivamente, el riesgo de muerte fetal intrauterina no es más grande que el de la población obstétrica general; igualmente, que la frecuencia total y la severidad de las patologías perinatales es menor.

Otros factores que determinan un mal pronóstico maternofetal, según Pedersen, son: (1)

- Pielonefritis clínica

- Acidosis química o cetoacidosis grave

- Preeclampsia-eclampsia

- Negligencia (no siguen el plan de tratamiento o consultan tardíamente)

Tabla 4

DIAGNóSTICO DE DMG CON PTOG DE 100 Y 75 G

\begin{tabular}{|l|c|c|}
\hline & $\begin{array}{c}\text { Carpenter y } \\
\text { Coustan }(\mathbf{1 0 0} \mathbf{g})\end{array}$ & $\begin{array}{c}\text { OMS y } \\
\text { otros } \mathbf{7 5} \mathbf{~ g})\end{array}$ \\
\hline Ayunas & 95 & 95 \\
1 hora & 180 & 180 \\
2 horas & 155 & 155 \\
3 horas & 140 & - \\
\hline
\end{tabular}


Tabla 5

CRITERIOS PARA EL DIAGNÓSTICO DE DM

1. Síntomas de diabetes (poliuria, polidipsia y pérdida de peso) acompañadas de una glucosa plasmática a cualquier hora $\geq 200 \mathrm{mg} / \mathrm{dl}$.

2. Glucosa plasmática en ayunas $\geq 126 \mathrm{mg} / \mathrm{dl}$. Ayunas = no ingesta de alimentos al menos durante ocho horas.

3. Glucosa plasmática a las dos horas $\geq 200 \mathrm{mg} / \mathrm{dl}$ durante una PTOG con $75 \mathrm{~g}$ disueltos en agua.

Por tanto, hay tres formas de diagnosticar la DM y cada una debe ser confirmada en un día diferente por cualquiera de los tres métodos, en ausencia de hiperglicemia severa con descompensación aguda. Los valores normales para la glicemia en ayunas, se han establecido en $110 \mathrm{mg} / \mathrm{dl}$; la razón para ello es la pérdida de la primera fase de secreción de insulina a partir de esta cifra. Las categorías para la glicemia en ayunas quedan establecidas así:

\section{Glicemia normal $\longrightarrow<110 \mathrm{mg} / \mathrm{dl}$}

Hiperglicemia en ayunas $\longrightarrow$ entre 110 y $125 \mathrm{mg} / \mathrm{dl}$ Diagnóstico provisional de DM $\longrightarrow \geq 126 \mathrm{mg} /$ dl (debe ser confirmado)

Para la PTOG, las categorías son:

Glicemia normal $\longrightarrow$
poscarga

Disminución de la tolerancia a la glucosa entre 140 y $199 \mathrm{mg} / \mathrm{dl}$, dos horas poscarga

Diagnóstico provisional de DM

$\mathrm{mg} / \mathrm{dl}$ (debe ser confirmado)

Pruebas para DM en la población asintomática: la prevalencia de DM no diagnosticada en la población general, puede llegar hasta el $50 \%$ de la población diabética; de ahí, que la DM no diagnosticada es una condición importante y su diagnóstico temprano y su tratamiento adecuado, disminuirá la prevalencia de complicaciones. Por tanto, se recomienda realizar pruebas en poblaciones asintomáticas de alto riesgo, así:

En mayores de 45 años y luego cada tres años si son normales

Se harán pruebas a una edad más temprana o con mayor frecuencia en:

Obesos: $\geq 120 \%$ de peso ideal o con IMC $\geq 27 \mathrm{~kg} / \mathrm{m}^{2}$ Tener un familiar con DM en primer grado

Ser miembro de grupos étnicos de alto riesgo (ver antes) Mujeres que hayan tenido hijos con más de $4.500 \mathrm{~g}$ al nacer o que hayan presentado DMG previa.

Ser hipertenso $(\geq 140 / 90)$

Tener HDL $\geq 35 \mathrm{mg} / \mathrm{dl}$ y/o TG $\geq 250 \mathrm{mg} / \mathrm{dl}$

Tener previamente disminución de la tolerancia a la glucosa en ayunas o poscarga
El manejo de la DM durante el embarazo debe realizarse en un nivel de atención terciaria, siendo el deber de los niveles de atención primaria el diagnóstico precoz, su manejo inicial y la remisión oportuna. Dicho manejo requiere de un enfoque interdisciplinario, puesto que involucra aspectos farmacológicos, educativos, nutricionales, sociales, psicológicos y familiares. Para reducir significativamente la mortalidad materno-fetal, el equipo de trabajo debe incluir: un médico obstetra, un diabetólogo o médico entrenado en diabetes, un neonatólogo, una enfermera educadora, una nutricionista y un psicólogo o trabajador social. Igualmente, la paciente y su familia deben tomar parte activa en el tratamiento, siendo la motivación y la educación elementos fundamentales para su integración.

\section{Objetivos del manejo de la DM durante el embarazo}

7.1 Detección de la embriopatía diabética: la principal causa de morbimortalidad perinatal en las pacientes con DM pregestacional son las malformaciones congénitas fetales, con una incidencia entre 7,5 y 14,9\% (7 a 15 veces más frecuente que en los embarazos normales). Las alteraciones morfológicas más frecuentes son las cardiovasculares (transposición de grandes vasos, defectos del septum ventricular y auricular) y neurológicas (anencefalia, holoprocencefalia y defectos del cierre del SNC). $(1,3,5)$.

La paciente diabética que desea embarazarse, debe recibir una adecuada asesoría preconcepcional. Ahora, la detección de anomalías estructurales (producidas por la hiperglicemia presente durante las primeras ocho semanas de gestación período crítico) incluye:

- Medición de la HbA1C de la paciente 4 - 6 semanas

- posconcepción: niveles superiores a $8,5 \%$ se asocian a un $20-25 \%$ de probabilidades de desarrollo de anomalías fetales versus $2-3,5 \%$ cuando las cifras de HbA1C son normales (3-4).

- Ecografía vaginal entre las 8 - 10 semanas de amenorrea, sobretodo en aquellas pacientes con cifras anormales de HbA1C. Algunos defectos se diagnostican fácilmente por este tiempo (anencefaliaholoprocencefalia).

- Detección de alfafetoproteína sérica materna (AFPSM) a las 16 semanas de gestación. Normalmente esta AFPSM está disminuida en las pacientes diabéticas y suele indicar la necesidad de realizar estudio genético mediante amniocentesis/cordocentesis.

- Ecografía de tercer nivel entre las 18 - 20 semanas de gestación, para detectar malformaciones fetales no visualizadas en ecos iniciales.

- Ecocardiograma fetal entre las 24 - 28 semanas de gestación

7.2 Visitas prenatales: de óptima calidad, realizándolas cada 15 días hasta la semana 24 y de ahí en adelante cada semana, solicitando los siguientes paraclínicos: (1)

Citoquímico de orina y urocultivo: las infecciones urinarias son más frecuentes durante la gestación y son la primera causa de descompensación en la 
paciente diabética. Se solicitarán en la primera consulta prenatal y luego en forma mensual.

BUN - creatinina y ácido úrico: como indicadores mínimos de función renal, en forma trimestral. Perfil lipídico: inicial, a las 24 - 26 semanas de gestación y al final de la misma

- HbA1C: a las 4 - 6 semanas de la concepción y luego cada mes, para analizar la calidad del control metabólico, manteniéndola por debajo de 7,2\%.

- Monitoreo glicémico y pruebas de bienestar fetal (ver más adelante)

- Fundoscopia y EKG: en DM pregestacional con compromiso vascular

- Ecografías seriadas: detectar malformaciones y evaluar crecimiento fetal

7.3 Estricto control metabólico materno: los pilares del tratamiento de la diabetes gestacional son:

El control estricto de las glicemias durante el embarazo, y

- La detección precoz de los factores de riesgo y/o agravante s del estado metabólico $(1,19)$

Los objetivos del control metabólico son:

Evitar las hipoglicemias sintomáticas

Conservar las siguientes cifras de glicemia en plasma:

En ayunas ------ $>65$ a $<105 \mathrm{mg} / \mathrm{dl}$ (ideal $<95 \mathrm{mg} / \mathrm{dl}$ )

Preprandial ------ > $70-100 \mathrm{mg} / \mathrm{dl}$

Una hora posprandial ------ > $<140 \mathrm{mg} / \mathrm{dl}$ (ideal $<130$ $\mathrm{mg} / \mathrm{dl}$ )

Dos horas posprandial ------ $><120 \mathrm{mg} / \mathrm{dl}$

Cetonuría negativa

HbA1C y/o fructosamina: normal baja

Ahora, para lograr los objetivos del control metabólico se debe formular un plan terapéutico que incluya: dieta, ejercicio, insulina (si es necesaria) y el manejo de las complicaciones.

Dieta: es la clave o apoyo principal de la terapéutica en mujeres con diabetes gestacional. La ganancia de peso es necesaria para el adecuado crecimiento fetal y varía entre 8 y $12 \mathrm{~kg}$, dependiendo del peso previo de la paciente. Recordar que la paciente nunca debe perder peso durante la gestación, ya que ello conlleva a movilización de grasas, lipólisis y sustancias que atraviesan la placenta y que tienen efectos teratogénicos pontenciales (1). La dieta debe aportar un promedio de $30-35 \mathrm{cal} / \mathrm{kg}$ en pacientes de peso promedio y $25 \mathrm{cal} / \mathrm{kg}$ en pacientes obesas, fraccionada en tres comidas principales y tres refrigerios, haciendo énfasis en el refrigerio de las 22:00 - 23:00 horas, para evitar la hipoglicemia materna en la madrugada (vaso con leche y galletas) $(15,20)$. Recordar que un gramo de proteínas o de $\mathrm{H}$ de $\mathrm{C}$ aportan cuatro calorías y que un gramo de grasas proporcionan nueve calorías. Se ha visto que limitando la toma de $\mathrm{H}$ de $\mathrm{C}$ a 35 - $45 \%$ del total de calorías, se controla más adecuadamente la glicemia posprandial (9).

Ejercicio regulado y adecuado es beneficioso y seguro; libera epinefrina, la cual aumenta la captación de glucosa por la célula y aumenta la sensibilidad de los receptores, manteniendo estables los niveles de glicemia. Se debe caminar entre 10 y 20 minutos después de cada comida principal.

- Insulina: los requerimientos de insulina aumentan progresivamente durante la gestación. En el primer trimestre son frecuentes las hipoglicemias severas y disminuyen los requerimientos de insulina. Durante el segundo trimestre, se presenta un incremento leve mensual; en el tercer trimestre pueden presentarse hipoglicemias nocturnas y los requerimientos de insulina aumentan en un $50-100 \%$ por encima de la basal. Los hipoglicemiantes orales están contraindicados para el control de la diabética embarazada, ya que atraviesan la placenta y originan hipoglicemias fetal es severas y malformaciones $(1,5)$. Deben recibir insulina:

- Las DM1 y las DM2 siempre que se embaracen

- Entre las pacientes con DMG, el grupo A2 de White modificado por Freinkel (glicemia en ayunas entre 105 y $129 \mathrm{mg} / \mathrm{dl}$ ) y el grupo B (glicemia en ayunas > 130 $\mathrm{mg} / \mathrm{dl}), \mathrm{y}$

Aquellas pacientes con DMG (15 - 22\%), quienes con un régimen estricto de dieta y ejercicio durante dos semanas, presenten glicemia en ayunas $\geq 105 \mathrm{mg} / \mathrm{dl}$ o glicemias dos horas posprandial $\geq 120 \mathrm{mg} / \mathrm{dl}$, en dos o más ocasiones. Estas pacientes se benefician enormemente de la insulina, disminuyendo las tasas de morbilidad fetal y neonatal (macrosomía, parto traumático, etc).

La insulina utilizada durante el embarazo debe ser preferiblemente humana, la cual disminuye la formación de anticuerpos anti-insulina, los cuales pasan la placenta y contribuyen al aumento de la insulina libre fetal y por ende a la macrosomía fetal y a la hipoglicemia neonatal. Se pretende mantener la glicemia por debajo de 95,140 y $120 \mathrm{mg} / \mathrm{dl}$ en ayunas, una y dos horas posprandial respectivamente $(3,9,13$, 23). En 1997 aparece en el mercado la insulina Lispro, la cual presenta las siguientes ventajas: una velocidad de absorción mayor, por 10 cual se debe colocar inmediatamente antes de las comidas y una duración de acción menor, lo que disminuye la posibilidad de hipoglicemias posprandiales y la necesidad de las entrecomidas; esta insulina lispro, puede ser sustituida 1: 1 por insulina regular. En la tabla 6, $(3,24)$ se puede observar la acción de las diferentes insulinas.

Los esquemas recomendados de insulina en la diabética gestacional, son: $(1,4)$

1. Según el perfil glicémico antes del desayuno, se debe comenzar con una dosis pequeña de NPH $(5-10 \mathrm{u})$ antes del desayuno, con una mezcla de NPH e insulina regular (15 u de NPH más 5 u de regular) antes del desayuno o con una dosis pequeña de insulina regular $(10 \mathrm{u})$ antes de la comida que causa la elevación posprandial, ajustando la dosis de acuerdo con el automonitoreo, así:

Si la glicemia posdesayuno es $>140 \mathrm{mg} / \mathrm{dl}$, se debe colocar o aumentar la insulina cristalina antes del desayuno.

Si la glicemia dos horas posalmuerzo es $>140 \mathrm{mg} / \mathrm{dI}$, se debe aumentar la NPH antes del desayuno. 
Tabla 6

ACCION DE LAS DIFERENTES INSULINAS

\begin{tabular}{|l|r|l|l|}
\hline Insulina & Inicio & \multicolumn{1}{c|}{ Pico } & \multicolumn{1}{c|}{ Duración } \\
\hline $\begin{array}{l}\text { Cristalina o } \\
\text { regular } \\
\text { humana }\end{array}$ & $30-60$ min. & 2-3 horas & $4-6$ horas \\
NPH humana & $2-4$ horas & $6-10$ horas & 6-18 horas \\
$\begin{array}{l}\text { Ultralenta } \\
\text { humana }\end{array}$ & $6-10$ horas & Mínimo & 20-30 horas \\
Lispro & 15 minutos & $30-90$ min. & $3-4$ horas \\
Cristalina IV & Inmediato & 5 minutos & 25 minutos \\
\hline
\end{tabular}

Si la glicemia dos horas poscomida es $>140 \mathrm{mg} / \mathrm{dl}$, se debe colocar o aumentar la insulina cristalina antes de la comida.

- Si la glicemia en ayunas es $>105 \mathrm{mg} / \mathrm{dl}$, se debe colocar o aumentar la NPH antes de la comida.

2. La dosis típica de insulina es de $0,7 \mathrm{u} / \mathrm{kg} /$ día en el primer trimestre, debiendo ser incrementada al progresar el embarazo $(0,9 \mathrm{u} / \mathrm{kg} / \mathrm{día}$ en la mitad y $1 \mathrm{u} / \mathrm{kg} /$ día al final). Si se requieren distribuciones de insulina, se harán así:

\begin{tabular}{lll} 
& AM & PM \\
NPH & $2 / 3$ & $1 / 2$ \\
Cristalina & $1 / 3$ & $1 / 2$ \\
\cline { 2 - 3 } Dosis total & $2 / 3$ & $1 / 3$
\end{tabular}

Se deben mezclar la insulina cristalina y la NPH en la misma jeringa y aplicarlas 30 minutos antes de desayuno y comida. Cada tres días se reajusta la dosis de ellas, según el perfil glicémico, aumentando o disminuyendo el $20 \%$ de acuerdo con el pico máximo de acción de la NPH (18 horas) y de la insulina regular (6 horas).

7.3 Monitoreo de la glicemia: solo ameritan hospitalización las pacientes de difícil manejo, las que tienen diagnóstico reciente $o$ las que presentan complicaciones. Se debe insistir en el automonitoreo con tiras reactivas para lectura visual o con reflectómero, diariamente y a distintas horas; la paciente anotará en su libreta de automonitoreo los resultados de la glicemia, según el siguiente esquema: primer día glicemia en ayunas, segundo día glicemia dos horas posdesayuno, tercer día glicemia dos horas posalmuerzo, cuarto día glicemia dos horas poscomida, quinto día glicemia en ayunas y así sucesivamente. Este esquema nos ofrece una información adecuada del control metabólico. En las pacientes hospitalizadas se debe determinar la glicemia en ayunas, dos horas posdesayuno, dos horas posalmuerzo, dos horas poscomida y a las tres - cuatro de la madrugada (esta última con el fin de diagnosticar la hipoglicemia nocturna y el efecto Somogy).
7.4 Detección precoz de los factores de riesgo y/o agravantes del estado metabólico: controlado el ambiente metabólico, es responsabilidad del obstetra detectar los estados patológicos que afecten los resultados de morbilidad maternofetal, como son: $(1,3-5,9)$.

Preeclampsia - eclampsia: se asocia al 25\% de las diabéticas gestacionales, afecta los índices de mortalidad fetal y favorece el aumento de morbilidad por prematurez.

Hidramnios: se asocia al 19 - 25\% de los embarazos diabéticos, consecuencia lógica de los estados de hiperglicemia materno-fetal, que conducen a una mayor producción urinaria fetal.

Prematurez: tan temida como lamacrosomía, ya que va acompañada del síndrome de dificultad respiratoria (SDR), causa principal de la morbimortalidad perinatal; este SDR se presenta entre el 5 y $30 \%$ de los hijos de madres diabéticas y es debido al efecto inhibitorio de la insulina sobre las enzimas que intervienen en la síntesis y secreción de surfactante; tanto la inmadurez como la vía del parto influyen en la aparición del síndrome. La incidencia de la prematurez es del $19-40 \%$ y es secundaria a complicaciones como: preeclampsia - eclampsia, hidramnios, macrosomía, insuficiencia placentaria, RPMO e infección intrauterina.

Infecciones: principalmente las de vías urinarias; favorecen la prematuridad, la RPMO y trastornan el control metabólico.

Alteraciones del crecimiento fetal (macrosomía y $R C I U)$ : desde la séptima semana el feto sintetiza insulina y su producción aumenta por encima de los valores normales; en respuesta a la hiperglicemia predominante, la actividad lipogénica de la hormona se traduce en macrosomía fetal. Esta es la complicación fetal más frecuente y más temida de las madres con DMG y es la principal causa de morbilidad neonatal y de complicación intraparto $(1,3-4,25)$. Se presenta entre el $15-45 \%$ de los embarazos diabéticos y aumenta la frecuencia de trauma fetal intraparto, los índices de hipoglicemia, las tasas de cesárea y la hemorragia posparto.

Las pacientes obesas tienen mayor riesgo de desarrollar un bebé macrosómico (5 - 14\% versus 1,7\%) según Spellay citado por Restrepo (1). Si bien el riesgo de desarrollar macrosomía puede alterarse desde el período preconcepcional, es necesario que la gestante alcance el control metabólico antes de la semana 32, para reducirla del $44 \%$ en pacientes de control pobre al $11 \%$ en las pacientes con buen manejo.

Por tanto, todas las pacientes diabéticas embarazadas deben someterse a ecografías seriadas cada cuatro semanas a partir de la semana 20 de gestación, para monitorizar el crecimiento fetal; al final del embarazo, la estimación clínica y la evaluación ecográfica habitualmente coinciden en el diagnóstico de feto grande (4). Los hijos de madres con DMG tienen aumentado el riesgo de obesidad, intolerancia a la glucosa y diabetes en la adolescencia tardía y la adultez joven $(9,26)$.

7.5 Pruebas de bienestar fetal: existe consenso en que las pacientes con DM insulinodependiente y que las pacientes con DMG que presentan hiperglicemia en ayunas $\quad{ }^{3}$ $105 \mathrm{mg} / \mathrm{dl}) \quad \mathrm{y} / \mathrm{o}$ posprandial $(\geq 120 \mathrm{mg} / \mathrm{dl})$, deben ser 
sometidas a pruebas de bienestar fetal en las últimas 8 -10 semanas de gestación, debido al incremento de muerte fetal intrauterina y de muerte neonatal $(4,9,26)$.

El inicio del control anteparto depende de la gravedad y estabilidad de la diabetes materna. Así, el automonitoreo de movimientos fetales se debe llevar a cabo a partir de la semana 28, cada dos días, posprandial y durante 30 minutos. Arias sugiere que las pacientes con diabetes inestables insulinodependientes $\mathrm{y} / \mathrm{o}$ de difícil manejo, deben monitorizarse desde la semana 28, las insulinodependientes controladas desde la semana 34 y las diabéticas gestacionales desde la semana 36 (4). Parece haber equivalencia entre las pruebas de bienestar fetal, siempre que se elija un intervalo adecuado de vigilancia, así: NST dos veces/semana, CST semanal, perfil biofísico semanal o doppler de cordón semanal; entre el CST y el NST se prefiere el primero, ya que detecta más precozmente la presencia de compromiso fetal.

47.6 Decidir el momento oportuno del parto y la vía adecuada del mismo: $(1,3-5,9,26)$ la decisión del momento del parto debe individualizarse en cada paciente. En las pacientes controladas metabólicamente y sin ninguna patología sobreagregada el embarazo puede llegar al término y elegirse preferencialmente la vía vaginal; no se debe dejar avanzar el embarazo más allá de la semana 40 por el aumento de la tasa de mortalidad perinatal. Ahora, en la paciente diabética descompensada solo se debe considerar la presencia de madurez pulmonar fetal por encima de las 38 semanas de gestación o con valores de fosfatidilglicerol positivos (> $3 \%$ en LA obtenido por vía vaginal o por amniocentesis), relación $\mathrm{L} / \mathrm{E} \geq 3: 1$ o test de Clements positivo; el valor predictivo positivo de éste (pulmón fetal maduro) es del 99 - 100\%, variando el valor predictivo negativo (pulmón fetal inmaduro) entre el 10 y $92 \%$ según el estudio.

Según la cuarta conferencia internacional de trabajo en diabetes gestacional, la presencia de DMG no constituye por si misma una indicación para cesárea o para parto antes de las 38 semanas de gestación; igualmente presentan datos que indican que los índices altos de cesárea son debidos a la asociación de complicaciones obstétricas. Por otro lado, la prolongación de la gestación por encima de las 38 semanas incrementa el riesgo de macrosomía (9). El manejo de la macrosomía es controvertido: la cesárea está justificada cuando el peso fetal calculado es $\geq$ $4.500 \mathrm{~g}$. Algunos autores recomiendan igualmente el parto por vía alta cuando el peso fetal es > de $4.000 \mathrm{~g}$, ya que según ellos, el paniculoadiposo de los hombros y el tronco pueden hacer que estos sean mayores que la cabeza, favoreciéndose la distocia de hombros; otros no defienden la cesárea en fetos entre 4.000 y $4.500 \mathrm{~g}$, debido al margen de error en el cálculo del peso fetal por ecografía y la baja incidencia de lesiones fetales, recomendando la inducción dos o tres semanas antes de la fecha probable del parto, siempre y cuando el cuello sea favorable (Bishop $\geq 6$ ) y haya madurez pulmonar fetal (4). Para otros autores, la macrosomía ( $\geq 4.000 \mathrm{~g}$ ) es indicación absoluta para cesárea.

No es necesario someter a cesárea a todas las pacientes diabéticas insulinodependientes, y aunque existen múltiples indicaciones para realizarla, más del $50 \%$ de estas pacientes pueden tener un parto vaginal sin riesgo.
Ahora, en pacientes con daño vascular, HTA y/o preeclampsia-eclampsia, antecedentes de mortinatos y RCIU, se debe considerar la terminación del embarazo, analizando cada caso y garantizando la madurez pulmonar fetal, realizando cesárea cuando haya indicaciones obstétricas y/o fetales.

7.7 Manejo intraparto - Esquemas del manejo de la insulina: el objetivo del manejo metabólico durante el trabajo de parto ( $\mathrm{T}$ de P) es mantener la glicemia entre $80-110 \mathrm{mg} / \mathrm{dl}$. (3)

Inducción o T de $P$ en pacientes con DMG controlada con dieta: debe instaurarse una infusión de DAD 10\%, a raz6n de 5 a $10 \mathrm{~g} /$ hora para evitar las hipoglicemias maternas.

Inducción o T de $P$ en pacientes con DMG controlada con insulina o con DM pregestacional: se colocará una infusión combinada de insulina y dextrosa, tal como se observa en la tabla 7. (3)

En el parto por cesárea:

En pacientes con DMG controlada con dieta: ideal realizarla en las horas de la mañana, llevando a la

\section{Tabla 7 \\ CONTROL DE LA GLICEMIA MATERNA INTRAPARTO}

1. Infusión continua de insulina:

No administrar la dosis matinal de insulina

- Se coloca una infusión continua de DAD 5\% a razón de $100 \mathrm{cc} /$ hora a lo largo del T de $\mathrm{P}$.

- Se coloca una infusión continua de 50 u de insulina cristalina en $500 \mathrm{cc}$ de solución salina, a una velocidad de $0,5 \mathrm{u} /$ hora.

- Iniciar oxitocina si es necesario

- Monitorizar glicemia materna cada hora

- Ajustar la infusión de insulina, según la glicemia, así:
Glicemia en plasma (mg/dl)

$$
\begin{gathered}
<80 \\
80-100 \\
101-140 \\
141-180 \\
181-220 \\
>220
\end{gathered}
$$

Rata de infusión (unidades/hora)

No aplicar insulina

0,5

1,0

1,5

2,0
2. Infusión intermitente de insulina:

- Se administra la mitad de la dosis de insulina matinal, en forma de NPH

Se coloca una infusión continua de DAD 5\% a 100 cc/hora

Iniciar oxitocina si es necesario

Monitorizar la glicemia materna cada hora

Administrar bolos de insulina regular de 2 a 5 unidades, manteniendo la glicemia materna entre $80-110 \mathrm{mg} / \mathrm{dl}$. 
paciente a cirugía con goteo de solución salina, para evitar la hipoglicemia neo natal.

En pacientes con DMG que reciben insulina o en pacientes con DM pregestacional con un buen control: se administra la mitad o cuarta parte de la dosis matinal de insulina, en forma de NPH. La infusión de insulina descrita se suspende una vez se produce el nacimiento, tanto por vía vaginal como por cesárea. La infusión de glucosa se continúa hasta la comida siguiente cuando el parto es por vía vaginal o por espacio de 24 horas (o hasta iniciar la dieta normal) en caso de cesárea. En las primeras 24 horas no se requiere insulina generalmente, pero debe determinarse la glicemia y cuerpos cetónicos cada cuatro - seis horas. La administración de insulina subcutánea se debe iniciar cuando las glicemias en ayunas son mayores de $110 \mathrm{mg} / \mathrm{dl}$ o cuando son mayores de $180 \mathrm{mg} / \mathrm{dl}$ durante la administración de suero glucosado, según esquema establecidos.

En las pacientes con DMG, luego del parto, hay normalización de las glicemias; sin embargo, se deben determinar glicemias cada 12-24 horas durante los primeros días del puerperio, para detectar a las pacientes en quienes persiste la diabetes después del parto. A las seis semanas posparto, la paciente debe someterse a una PTOG con $75 \mathrm{~g}$, tomando muestras en ayunas y dos horas posprandial, con el fin de ser reclasificada como diabética, intolerancia a la glucosa en ayunas, intolerancia a la glucosa o normal, tal como se anotó antes (tabla 5).

\section{BIBLIOGRAFÍA}

1. Restrepo O. Enfoque y manejo de la embarazada diabética. Revista Colombiana de Obstetricia y Ginecología. Abril - Junio, 1992; 43(2): 97-108.

2. White P, Titus RS, Joslin EP. Prediction and prevention of late pregnancy accidents in diabetes. Am J Med Sci. 1939; 198: 482- 492.

3. Moore TR. Diabetes in pregnancy. En: Creasy Resnik. MatemalFetal Medicine. 4th edition. USA: W.B. Saunders Company, 1999; 964-995.

4. Arias F. Diabetes y Embarazo. En: Guía práctica para el embarazo y el parto de alto riesgo. Segunda edición. Madrid: Mosby / Doyma libros, 1994; 284-303.

5. Parra MO, Peralta MT. Diabetes y Gestación. En: Náñez H, Ruiz Al. Obstetricia y Perinatología. Primera edición. Santafé de Bogotá: Lito Camargo, 1999; 637-647.

6. National Diabetes Data Group. Classification and diagnosis of diabetes mellitus and other categories of glucose intolerance. Diabetes, 1979; 28: 1039-1057.

7. World Health Organization. Diabetes Mellitus: report of a WHO Study Group. Geneve, World Health Org. 1985; 727.

8. Report of the Expert Committee on the Diagnosis and Classification of Diabetes Mellitus. Diabetes Care. July, 1997; 20(7):1183- 1197.

9. Summary and Recommendations of the Fourth international Workshop - Conference on Gestational Diabetes Mellitus. Diabetes care. August, 1998; 21(suppl 2): BI21-BI67.

10. Kjos SL. Peters RK, Xiang A, et al. Predicting future diabetes en Latino women with gestational diabetes. Utility of early postpartum glucose tolerance testing Diabetes, 1995; 44: 586.

11. O' Sullivan JB, Mahan CM. Criteria for the oral glucose tolerance test in pregnancy. Diabetes May - Jun, 1964; 13(3): 278-285.

12. Diabetes and pregnancy. ACOG. Technical Bulletin. 1994; 200.

13. Freinkel N, Josimovich J. Conference Planning Committee: American Diabetes Association Workshop - Conference on Gestational Diabetes: sumrnary and recornrnendations. Diabetes Care. 1980; 3; 499-501.

14. Freinkel N. Summary and recommendations of the second International Workshop - Conference on Gestational Diabetes Mellitus. Diabetes, 1985; 34(suppl 2): 123-126.
15. Metzger BE. The organizing committee: summary and recommendations of the third International Workshop - Conference on Gestacional Diabetes Mellitus. Diabetes, 1991; 40(suppl 2): 197201.

16. Carpenter MW, Coustan DR. Criteria for screening tests for gestational diabetes. Am. J. Obstet Gynecol. 1982; 144: 763-773.

17. Deerochanawong $\mathrm{C}$, Putiyanum $\mathrm{C}$, Wongsuryrat $\mathrm{M}$ et al. Comparison of National Diabetes Data Group and World Health Organization Criteria for detecting gestational diabetes mellitus.Diabetología, 1996; 39: 1070-1073.

18. Pedersen J. La diabética gestante y su recién nacido. Problemas y tratamiento. Barcelona, Salvat editores. 1981; 278.

19. Gabbe SO. Definition, detection and management of gestational diabetes. Obstet Gynecol. 1986; 67(1): 121-125.

20. Algert $S$ et al. Moderate caloric restriction in obese women with gestational diabetes. Obstet Gynecol. Apr, 1985; 65(4): 487-491.

21. Freinkel N, Metzger. BE, Phelps RL et al. Gestational Diabetes Mellitus. Heterogeneity of maternal age, weight, insuline secretion, HLA antigens, and islet cells antibodies and the impact of maternal metabolism on pancreatic B-cell and somatic development in the offspring diabetes. Jun, 1985; 34(suppl 2): 1-7.

22. American Diabetes Association Position Statement. Gestational Diabetes Mellitus. Diabetes Care. January, 1995; 18 (suppl 1):24-25.

23. Deckert T. The inmunogenicity of new insulins. Diabetes. Jun, 1985; 34 (suppl 2): 94-96.

24. Villegas P. Que hay de nuevo en diabetes mellitus. En: Actualización en Medicina Interna. Primera edición. Medellín - Colombia: Imprenta U. de A., 1998; 275-287.

25. Widness JA, Cowett RM, Constan DR et al. Neonatal morbidities in infants of mothers with glucose intolerance in pregnancy. Diabetes. Jun, 1985; 34(suppl 2): 61-65.

26. American Diabetes Association. Gestational diabetes mellitus. Diabetes Care. January, 1999; 22(suppl 1): S74-S76. 